\title{
Phase Separation and Crystallization Induced by Electron Irradiation in Nanoscale $\mathrm{Fe}_{56.5} \mathrm{Mn}_{11} \mathrm{Cr}_{8.5} \mathrm{Ni}_{4} \mathrm{Si}_{10} \mathrm{C}_{10}$ Metallic Glass
}

\author{
Xiao-Yang Chen ${ }^{1} \cdot$ Shu-Guang Zhang ${ }^{1} \cdot$ Ming-Xu Xia $^{1} \cdot$ Jian-Guo Li $^{1}$
}

Received: 20 August 2015/Revised: 21 October 2015/Published online: 31 October 2015

(C) The Chinese Society for Metals and Springer-Verlag Berlin Heidelberg 2015

\begin{abstract}
A nanoscale $\mathrm{Fe}_{56.5} \mathrm{Mn}_{11} \mathrm{Cr}_{8.5} \mathrm{Ni}_{4} \mathrm{Si}_{10} \mathrm{C}_{10}$ metallic glass was irradiated by electron beam in a $200 \mathrm{kV}$ transmission electron microscope. Structure evolution in the metallic glass was investigated in situ during continuous irradiation, where phase separation was observed after irradiation for $5 \mathrm{~min}$ and crystallization was observed after $33.5 \mathrm{~min}$. Based on the analysis of irradiation effect, atomic displacement is believed to be the main reason for the structure rearrangement. Accumulation of atomic displacement increases the energy of the sample and promotes atomic diffusion during transformation. On the other hand, the large specific surface area of the sample also contributes to increasing free energy and atomic diffusion.
\end{abstract}

KEY WORDS: Metallic glass; Electron irradiation; Transmission electron microscopy; Phase separation; Crystallization

\section{Introduction}

Phase separation has been observed in various metallic glasses, such as Pd-, Fe-, Al-, La-, Ge-, Mg-, Cu-, Zr-, Tiand Y-based systems [1]. Phase separation turns the homogenous microstructure of metallic glasses into hierarchical composite. Metallic glasses with phase separation structure exhibit better plasticity than monolithic metallic glasses [2]. Phase separating metallic glasses can also be utilized to fabricate nanomaterials [3] and porous materials [4]. Apart from this, phase separation is considered to account for the high-density nucleation in devitrification of metallic glasses $[3,5]$. It is therefore important to investigate phase separation behavior in metallic glasses. By small

Available online at http://link.springer.com/journal/40195

Shu-Guang Zhang sgzhang@sjtu.edu.cn

1 School of Materials Science and Engineering, Shanghai Jiao Tong University, Shanghai 200240, China angle scattering, phase separation in Zr-based and Al-based metallic glasses has been studied in situ during annealing [6-8]. It is revealed that phase separation initiates in the supercooled liquid region, i.e., after glass transition and prior to crystallization. However, microstructure evolution cannot be presented in intuitive images by this method.

Transmission electron microscopy (TEM) is a useful characterization tool for in situ observation of structure evolution. The electron beam can stimulate and accelerate phase transformations in metastable materials [9]. For example, atomic rearrangement and crystallization can be driven by electron beam in amorphous carbon film [10], silica glass film [11] and $\mathrm{Cu}_{50} \mathrm{Zr}_{45} \mathrm{Ti}_{5}$ metallic glass [12]. Additionally, electron beam can provide a more controllable energy source for phase transformations, in terms of accelerate voltage and current density. Thus, structure rearrangement in metallic glasses, including phase separation and crystallization, can be investigated by TEM conveniently.

In this study, electron irradiation was carried out on a Febased metallic glass at ambient temperature. The structure evolution of the alloy was monitored and recorded in the form of morphology images and diffraction patterns. 


\section{Experimental}

$\mathrm{Fe}_{56.5} \mathrm{Mn}_{11} \mathrm{Cr}_{8.5} \mathrm{Ni}_{4} \mathrm{Si}_{10} \mathrm{C}_{10}$ (at\%) master alloy was prepared by induction-melting raw materials in argon (Ar) atmosphere. $\Phi 3 \mathrm{~mm}$ cylindrical sample was cast in copper mold. As-cast sample was a composite of crystalline and glassy phases. Glassy samples were collected through a chemical separation process, which is described more detail as follows: slice cut from the as-cast sample was soaked in a solution of $\mathrm{HF}: \mathrm{H}_{2} \mathrm{O}_{2}$ (3:50, in volume fraction) for hours at room temperature. Glassy phase was separated from the composite and reduced to small size. After etching, the solution containing residual amorphous phase was diluted with alcohol. The glassy phase was then attached to the carbon film for TEM (JEOL JEM-2100, $200 \mathrm{kV}$ ) observation. Composition of the glassy sample was examined by X-ray energy-dispersive spectroscopy (EDS) in TEM. During the morphology observation in TEM, the current density of electron beam is set at $10^{-9} \mathrm{~A} \mathrm{~cm}^{-2}$. The shot time of the image is marked in the picture. Each selected area diffraction pattern (SADP) was taken about $1.5 \mathrm{~min}$ after the corresponding morphology image.

\section{Results and Discussion}

The collected sample was checked by EDS before it was irradiated continuously. The result is listed in Table 1. It shows that the glassy phase is composed of $\mathrm{Fe}, \mathrm{Mn}, \mathrm{Cr}, \mathrm{Ni}, \mathrm{Si}$ and $\mathrm{C}$ elements. However, the carbon film inflects the results by highly increased $\mathrm{C}$ proportion. The ratio of other elements basically agreed with the master alloy. It confirms that the glassy phase is originated from the as-cast composite.

The evolution of the amorphous phase under electron irradiation is shown in Fig. 1. Figure 1a is the initial morphology of the glassy sample. The sample presents as irregular figuration and its shape is a quadrangle-like network. Thickness of the sample is fluctuant, which is reflected by different contrast. The uneven thickness reflects in two aspects. At macro level, there are several thick areas with dark contrast marked by arrows. At micro level, there is fine granular contrast in the sample, which is raised by the rough etching surface. SADP of the sample is shown in Fig. 1b. There is a halo ring $\left(R_{0}\right)$, predicting homogenous amorphous structure. Broad halo diffraction ring is the representative symbol of amorphous alloys [13, 14].

After irradiation for $5 \mathrm{~min}$, morphology of the sample changes slightly, as shown in Fig. 1c. Compared to the

Table 1 Composition of the glassy phase obtained by EDS (in at\%)

\begin{tabular}{llllll}
\hline $\mathrm{Fe}$ & $\mathrm{Mn}$ & $\mathrm{Cr}$ & $\mathrm{Ni}$ & $\mathrm{Si}$ & $\mathrm{C}$ \\
\hline 23.35 & 5.98 & 1.83 & 2.26 & 5.32 & 61.26 \\
\hline
\end{tabular}

initial state, the two-dimensional projected area decreases and the center hole becomes smaller, indicating that the sample shrinks under irradiation. The arrow marked areas are still thicker than others. Moreover, dark dots appear in the light matrix, presenting a typical morphology of phase separating microstructure. The decomposed dots present clear boundaries in thin areas, while in thicker areas they are hard to be distinguished. The corresponding SADP of the sample shows two halo rings $\left(\mathrm{R}_{1}\right.$ and $\left.\mathrm{R}_{2}\right)$ as shown in Fig. 2d.

After irradiation for $33.5 \mathrm{~min}$, the morphology and SADP are shown in Fig. 1e, f, respectively. The shape of the sample changes a lot after irradiation for $33.5 \mathrm{~min}$. It turns from quadrangle-like to triangle-like and the middle hole almost vanishes. It is obviously that the sample shrinks and its surface area decreases during irradiation. The relationship between surface area and the structural rearrangement will be discussed later. In the SADP, diffraction spots appear, suggesting the formation of crystals (Fig. 1f). These diffraction spots fall on two rings, marked as $\mathrm{R}_{\mathrm{c} 1}$ and $\mathrm{R}_{\mathrm{c} 2}$. The time interval between phase separation and crystallization is about half an hour. It is verified that phase separation initiates earlier than crystallization under electron irradiation.

Intensity profiles are helpful to identify delicate difference between SADPs. The radial intensity distribution profiles of the SADPs in Fig. 1b, d, f are plotted in Fig. 2. Horizontal axis is the diffraction radius, while vertical axis represents the relatively brightness of the pattern. The center diffraction spot is not shown in Fig. 2, where the radius begins from $2 \mathrm{~nm}^{-1}$. Intensity curves of SADPs at 0 , 5 and 33.5 min are drawn by solid curve, dashed curve and dot curve, respectively. The halo ring in SADP turns into broad peak on intensity curve. On the solid curve, $\mathrm{P}_{0}$ $\left(3.13 \mathrm{~nm}^{-1}\right)$ is the radial location of diffraction ring $\mathrm{R}_{0}$. On the dashed curve, $\mathrm{P}_{1}\left(3.35 \mathrm{~nm}^{-1}\right)$ is on the right of the initial peak $\mathrm{P}_{0}$ and $\mathrm{P}_{2}$ appears at further radial $\left(4.86 \mathrm{~nm}^{-1}\right)$. If the atomic arrangement in matrix phase and decomposed phase are significantly different, diffraction rings corresponding to matrix and decomposed phase would show different radius [15-17]. The emerging $R_{2}$ may be responsible for the decomposed phase. It is known that phase separation occurs between elements with positive heat of mixing $(\Delta H)$. Among the elements in this alloy, only $\Delta H$ of $\mathrm{Fe}-\mathrm{Mn}, \mathrm{Cr}-\mathrm{Mn}$ are non-negative [18], suggesting phase separation would occur between $\mathrm{Fe}, \mathrm{Cr}$ and $\mathrm{Mn}$. The decomposed phase is probably Mn-rich in composition. Phase separation is thought to be related to nanocrystallization behavior of metallic glasses and the crystals firstly nucleate in the decomposed phase [3, 5]. In the SADP of crystallization state (Fig. 1f), diffraction spots fell on two rings. On the corresponding intensity curve, the radial of the two rings are identified as $P_{c 1}$ at $4.82 \mathrm{~nm}^{-1}$ and $\mathrm{P}_{\mathrm{c} 2}$ at $6.92 \mathrm{~nm}^{-1} \cdot \mathrm{P}_{\mathrm{c} 1}$ is close to $\mathrm{P}_{2}$, suggesting the 

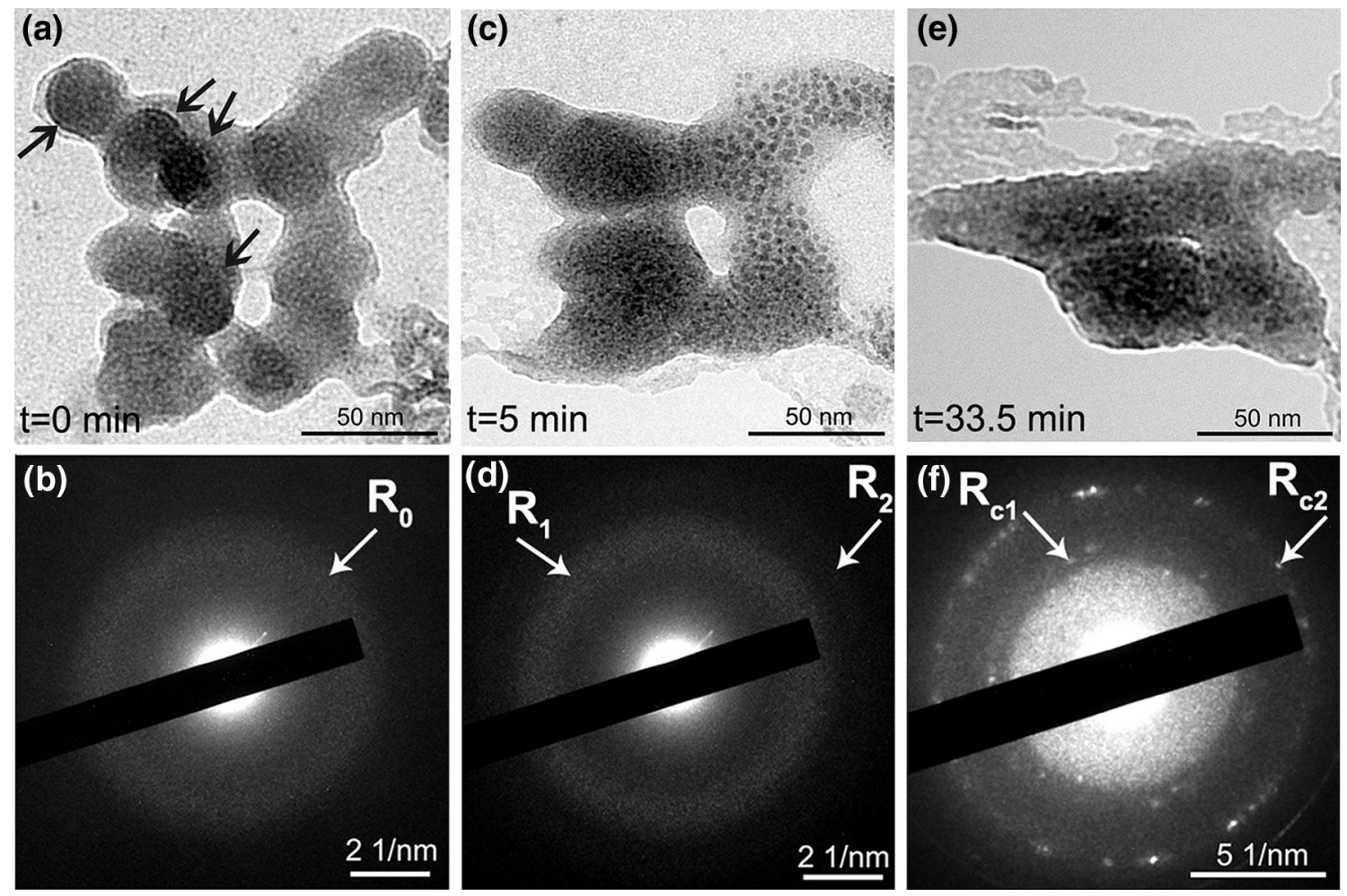

Fig. 1 Structure evolution in the amorphous phase in situ induced by electron irradiation $(200 \mathrm{kV})$ for different time and the corresponding diffraction patterns, where diffraction rings in every SADP are marked by arrow and named $R_{x}(x=0,1,2, \mathrm{c} 1, \mathrm{c} 2)$ : a, b 0 min; c, d 5 min; e, f $33.5 \mathrm{~min}$

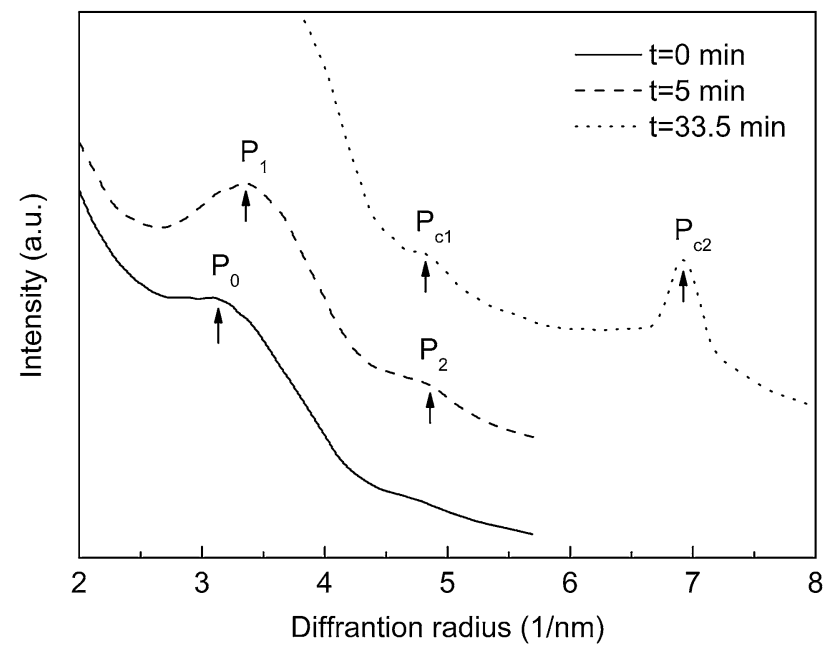

Fig. 2 Brightness distribution of SADP along the diffraction radius direction is expressed in terms of intensity profile. The solid curve, dashed curve and dot curve are the intensity profiles of the SADPs shown in Figs. 1b, d and f, i.e., corresponding to the sample irradiated for 0,5 and $33.5 \mathrm{~min}$, respectively. The peak of each diffraction ring is marked by $P_{x}(x=0,1,2, \mathrm{c} 1, \mathrm{c} 2)$

crystallization takes place in the decomposed phase. As for $\mathrm{P}_{\mathrm{c} 2}$, it may come from the same crystal phase with $\mathrm{P}_{\mathrm{c} 1}$ or it may be from other crystals.
As shown above, phase separation and crystallization are induced in the glassy sample during electron irradiation. Reason for structure evolution induced by electron irradiation is discussed from several aspects. The first issue is whether the phase transformation in the sample is caused by electron beam heating. Glass transition temperature of $\mathrm{Fe}-$ based metallic glasses is usually above $600 \mathrm{~K}$ [19-23]. Phase separation in metallic glasses occurs almost at the same temperature and crystallization temperature is even higher [24]. If electron beam can heat sample to that level, phase separation and crystallization can be caused. Electron beam heating relies on two conditions: high current density electron beam and poor thermal conduction. For example, temperature of a submicron Al-Si particle, which is shelled by a film of $\mathrm{Al}_{2} \mathrm{O}_{3}$, is raised $125 \mathrm{~K}$ by an electron beam of 7.3-17.6 $\mathrm{A} \mathrm{cm}^{-2}$ [25]. In the present study, the current density of electron beam is rather low $\left(10^{-9} \mathrm{~A} \mathrm{~cm}^{-2}\right)$ and the metallic glass is in good thermal contact with the carbon-supporting film. Thus, the electron beam is unable to heat the sample to glass transition and crystallization temperature. Phase transformations in this sample are not caused by temperature rising of electron irradiation.

Secondly, atomic displacement induced by irradiation is proposed the key issue in the electron-irradiation-induced 
phase transformations [12, 25, 26]. An atomic displacement forms when an atom is hit to leave its original position by electrons with high energy. An atomic displacement causes a free volume like defect and an anti-free volume like defect in metallic glass. The accumulation of defects increases the energy of the sample to trigger phase transformation. On the other hand, atomic displacement promotes atomic diffusion during structure evolution. Nagase et al. [27] provided the values of threshold voltages of electron beam to cause atomic displacement in different elements. Among these data, the threshold voltage for most metal elements is higher than $200 \mathrm{kV}$ (except for $\mathrm{Al}$ and $\mathrm{Mg}$ ). However, according to these threshold voltages, $200 \mathrm{kV}$ electron beam cannot induce atomic displacement in most alloys. It is conflict with the fact that atomic displacement is induced in $\mathrm{Cu}-\mathrm{Zr}-\mathrm{Ti}$ metallic glass by $200 \mathrm{kV}$ electron beam [12]. The threshold voltages of metal elements are probably overestimated. $200 \mathrm{kV}$ is possible to induce atomic displacement in the present sample. However, the current intensity of the beam in the present work is several orders of magnitude lower than that in previous cases where atomic rearrangement is induced in amorphous materials [10-12]. Accordingly, the density of induced defects in the present sample is comparatively low, requiring a long incubation time to accumulate defects for structure transformation.

Thirdly, the size and shape of the sample is related to structural rearrangement under electron irradiation. The irradiated sample is in irregular shape and in dimension of a few hundred nanometers. The sample shrinks and its surface area decreases with prolonging irradiation as shown in Fig. 2. It implies that, atoms, especially surface atoms, move a long distance during this period. Due to the tiny size and rough surface, the sample possesses a large value of specific surface area. The majority of atoms in the sample reside close to the surface, rendering a significant fraction of inherent imperfection with defects and vacancies. The surface imperfection raises the free energy of the sample to a higher level. As a result, the addition of a small quantity of defects induced by irradiation can increase the energy to trigger phase transformation. Also, it is known that atoms near surface migrate longer and more fiercely than inside atoms [11]. For samples with large specific surface area, the movement of surface atoms contributes most of atomic diffusion in structure transitions. Thus, it is concluded that the large specific surface area is the assistant reason for phase transformation induced by electron irradiation.

\section{Conclusions}

Phase separation and subsequent crystallization are induced by $200 \mathrm{kV}$ electron irradiation in the nanoscale $\mathrm{Fe}_{56.5} \mathrm{Mn}_{11} \mathrm{Cr}_{8.5} \mathrm{Ni}_{4} \mathrm{Si}_{10} \mathrm{C}_{10}$ metallic glass. Phase separation is observed after $5 \mathrm{~min}$ and crystallization after $33.5 \mathrm{~min}$. The phase transformation is attributed to the atomic displacement induced by irradiation and the large value of specific surface area of the sample.

Acknowledgments This work was financially supported by the National Natural Science Foundation of China (No. 51271119) and Doctoral Program of Higher Education of China (No. 20110073110005).

\section{References}

[1] D.H. Kim, W.T. Kim, E.S. Park, N. Mattern, J. Eckert. Prog. Mater. Sci. 58, 1103 (2013)

[2] X.H. Du, J.C. Huang, K.C. Hsieh, J.S.C. Jang, P.K. Liaw, H.M. Chen, H.S. Chou, Y.H. Lai, Adv. Eng. Mater. 11, 387 (2009)

[3] B. Radiguet, D. Blavette, N. Wanderka, J. Banhart, K.L. Sahoo, Appl. Phys. Lett. 92, 103126 (2008)

[4] J. Jayaraj, B.J. Park, D.H. Kim, W.T. Kim, E. Fleury, Scr. Mater. 55, 1063 (2006)

[5] S. Schneider, P. Thiyagarajan, W.L. Johnson, Appl. Phys. Lett. 68, 493 (1996)

[6] J.F. Löffler, W.L. Johnson, Appl. Phys. Lett. 76, 3394 (2000)

[7] X.L. Wang, J. Almer, C.T. Liu, Y.D. Wang, J.K. Zhao, A.D. Stoica, D.R. Haeffner, W.H. Wang, Phys. Rev. Lett. 91, 265501 (2003)

[8] J. Antonowicz, M. Kędzierski, E. Jezierska, J. Latuch, A.R. Yavari, L. Greer, P. Panine, M. Sztucki, J. Alloys Compd. 483, 116 (2009)

[9] R.F. Egerton, P. Li, M. Malac, Micron 35, 399 (2004)

[10] F. Börrnert, S.M. Avdoshenko, A. Bachmatiuk, I. Ibrahim, B. Buchner, G. Cuniberti, M.H. Rummeli, Adv. Mater. 24, 5630 (2012)

[11] P.Y. Huang, S. Kurasch, J.S. Alden, A. Shekhawat, A.A. Alemi, P.L. McEuen, J.P. Sethna, U. Kaiser, D.A. Muller, Science 342, 224 (2013)

[12] G.Q. Xie, Q.S. Zhang, D.V. Louzguine-Luzgin, W. Zhang, A. Inoue, Mater. Trans. 47, 1930 (2008)

[13] S. Báez, I. Betancourt, I.A. Figueroa, Acta Metall. Sin. (Engl. Lett.) 23, 401 (2010)

[14] G.W. Qin, N. Xiao, B. Yang, Y.P. Ren, W.L. Pei, X. Zhao, Acta Metall. Sin. (Engl. Lett.) 22, 415 (2009)

[15] A.A. Kündig, M. Ohnuma, D.H. Ping, T. Ohkubo, K. Hono, Acta Mater. 52, 2441 (2004)

[16] B.J. Park, H.J. Chang, D.H. Kim, W.T. Kim, K. Chattopadhyay, Phys. Rev. Lett. 96, 245503 (2006)

[17] N. Mattern, U. Kühn, A. Gebert, A. Schöps, T. Gemming, L. Schultz, Mater. Sci. Eng. A 449-451, 207 (2007)

[18] A. Takeuchi, A. Inoue, Mater. Trans. 46, 2817 (2005)

[19] A. Inoue, B.L. Shen, A.R. Yavari, A.L. Greer, J. Mater. Res. 18, 1487 (2003)

[20] S.J.Pang, T.Zhang, K. Asami, A. Inoue, Acta Mater. 50, 489 (2002)

[21] X.J. Gu, S.J. Poon, G.J. Shiflet, M. Widom, Acta Mater. 56, 88 (2004)

[22] K. Amiya, A. Urata, N. Nishiyama, A. Inoue, Mater. Trans. 45, 1214 (2004)

[23] Y. Wang, K. Xu, Q. Li, J. Alloys Compd. 540, 6 (2012)

[24] E.S. Park, J.H. Na, D.H. Kim, J. Appl. Phys. 108, 053515 (2010)

[25] T. Yokota, M. Murayama, J.M. Howe, Phys. Rev. Lett. 91, 265504 (2003)

[26] M.H. Li, Y.Q. Yang, B. Huang, X. Luo, W. Zhang, Y.X. Chen, M. Han, J.G. Ru, Acta Metall. Sin. (Engl. Lett.) 28, 147 (2015)

[27] T. Nagase, T. Sanda, A. Nino, W. Qin, H. Yasuda, H. Mori, Y. Umakoshi, J.A. Szpunar, J. Non-Cryst, Solids 358, 502 (2012) 